\title{
Knowledge Management and Modelling in Health Care Organizations: The Standard Operating Procedures
}

\author{
Vincenzo Della Mea, Marco Pittaro, and Vito Roberto \\ Dept. of Mathematics and Computer Science, University of Udine, Italy
}

\begin{abstract}
Standard Operating Procedures (SOP) in health care organizations are adopted for diagnostic procedures, laboratory examinations and similar, contain standardized prescriptions and are aimed at helping users to request services in the proper way. In the present paper, we describe a model and a software system for the management of SOP's in a hospital setup (HSOP), derived from similar approaches in the field of clinical protocols. In collaboration with medical experts, we analysed the Procedures Handbook of the City Hospital of Udine, Italy, in order to devise an abstract model for HSOP's and their management. The model has been described with a XML-schema and implemented with XML. A document workflow model has been extracted also, and realized into a workflow management system called HOPERA. Four categories of users have been considered, with different privileges on the HSOP management. HOPERA is a web application implemented on an open-source platform and includes user authentication, authoring features, archiving and retrieving HSOP's from an XML-native database, as well as workflow management tools. HOPERA is being tested in its operational environment, within the Udine City Hospital intranet system.
\end{abstract}

\section{Knowledge Management in Health Care}

By Knowledge Management (KM) we mean the set of methodologies and tools to manage the acquisition, organization, distribution and accessibility of the knowledge within an institution, to all the people who need it, whenever and wherever it is needed [1. In health care organizations, KM is playing a central role within a novel approach to the patient treatment and the quality control of diagnostic procedures. As a matter of fact, the mere contact between a medical doctor and a patient has evolved into a much more complex relation involving several professionals and experts, and associated to a large set of data, information and specialized medical knowledge.

Three basic processes have been individuated. Sense Making is aimed at keeping a health care organization abreast with the flow of information coming from the external world (biomedical research results, user demands, new laws and policies, etc). Such bursts of information are to be acquired, connected, analyzed in order to decide whether the organization itself is capable to address 
the novel needs. In case of positive answers, the second process - Decision Making - is activated to select the strategies and clinical actions to be undertaken. If the answers are negative, a Knowledge Creation step is to be devised to produce new knowledge and capabilities to bridge the gap that has emerged. In the present paper we focus on the last step: in particular, we propose a model for the management of Standard Operating Procedures (SOP's), by taking as a case study those adopted in the City Hospital of Udine, Italy. The model has brought practical results: an electronic representation of the SOP's, with a database enabling their storage and retrieval, an authoring system to assist the editing of HSOP's, and a workflow manager supporting the communications.

\section{$1.1 \quad$ Knowledge Creation and Learning}

Several kinds of knowledge may be individuated within an organization. The one owned individually and subjectively is called Tacit knowledge: quite often it is difficult to single out, but it is strategically relevant and many efforts are to be done to make it explicit and transferable. On the other hand, the Explicit Knowledge has been cast in a standard and structured form, so that can be readily found on public documents. Knowledge Creation (KC) is a process of social interaction involving individuals and adopting both kinds of knowledge; four ways to implement $\mathrm{KC}$ have been proposed [2].

- Socialization: Exchanging and sharing experiences through observation, listening, simulation. Tacit knowledge is transferred by acquiring technical capabilities;

- Externalization: Converting tacit to explicit knowledge by developing models, protocols, guidelines, procedures;

- Recombination: The existing explicit knowledge is reconfigured to synthesize new knowledge;

- Internalization: The acquisition process by the repeated execution of a task applying explicit knowledge, which brings additional tacit knowledge to an individual.

Learning processes are deeply intertwined with all $\mathrm{KC}$ steps, by providing a sort of dynamics controlling the KC strategies. We refer to the key concept of organizational learning, as proposed by Argyris and Schön [3]. It is the set of processes leading the organization to analyse critically its own successes and failures, to continuously revise its working procedures, to introduce and experiment novel solutions. We may say that an organization 'learns' whenever strategies and operational procedures - even those which appear consolidated and shared among multiple actors - change with time. Any individual or team in the organization is charged with a two-fold job: accomplishing his/her own specific tasks and learning from the same tasks in a sort of continuous knowledge-learning cycle. 


\section{The Standard Operating Procedures}

A key issue of KM in health care is developing standard models of behaviour (in its various forms); a few concepts are useful to this aim. As defined in the American Institute of Medicine Report [4, guidelines are "systematically developed statements to assist practitioner and patient decisions about appropriate health care for specific clinical circumstances" . Clinical guidelines and protocols identify the best practices derived from the scientific literature, through the so-called Evidence-Based Medicine approach, and thus commonly recognised as valid. A SOP is a set of specifications to point out: the purposes of an activity; what should be done and who is in charge to do it; the materials, tools and documents to be used; how they should be checked and registered.

At a first glance, SOP's may seem similar to guidelines; however, they combine two distinct kinds of knowledge on the process they model:

- general knowledge: the basic rules to carry out a task, often derived from guidelines and protocols;

- local knowledge: details on how the process is to be carried out in the local context, including resources, people, time, etc.

This makes every SOP a unique piece of knowledge, originated from the combination of universal and specific knowledge. Stated differently, both guidelines and SOP's indicate the sequence of actions recommended to accomplish definite health care operations in the optimal way. They include rigid rules: in so doing, put limitations to unjustified variations, and are intended to achieve uniform, standard behaviours. However, the sequence of actions described by a clinical guideline is in some way universal (with a few limitations), whilst SOP's are specifically developed for a site at a specified time.

\subsection{Representing Explicit Knowledge in Health Care}

The universal nature of clinical guidelines and protocols made them a good target for research regarding computer models and representation 7/9,10]11. 12. On the contrary, until the recent years, the SOP's appeared uniquely on paper, namely handbooks edited in a supposedly given standard format. In the medical domain, SOPs have been given a formal syntax only by two different research groups, by means of XML [56] . However, both are specific of laboratory procedures: the former in Pathology, the latter in chemistry laboratories. Now that authoring and communication tools are widespread due to the Internet, the need of an electronic support to the SOP's has become apparent, to make them available to a larger community; to make easier the revision cycles as well as the learning and training processes. In developing a method for modelling and representing SOP's, we took inspiration mostly from the researchers on guidelines and protocols.

We analyzed the state-of-the-art formalisms and concluded that, from our perspective, the Guidelines Element Model (GEM, [7]) is one of the most promising ones, in view of its performances in terms of completeness, flexibility, expressiveness, clarity and reusability. Based on the XML technology, GEM has been 
developed as a tool for representation, revision and translation during the whole life cycle of a guideline. It has been proposed as an international standard and already adopted as such by the American Society for Testing and Materials. However, as is, is not adequate for SOPs, thus we just started our study following the same approach.

\section{An Ontology for Hospital SOP's (HSOP's)}

Ontologies are considered as a key technology for Knowledge Management because they involve a consensus in the way a particular area of expertise is described. This consensus covers not only terminology, but also the way concepts and objects are organized and structured in the domain ([13]). In our preliminary work, we didn't try to obtain an inter-institution consensus on the procedure ontology, but rather to start from a case study, which we considered sufficiently heterogeneous to give insights on the structure and terminology for procedures.

In order to define a model of the HSOP's, we considered as a case study the laboratory and diagnostic test procedures adopted by the City Hospital of Udine, Italy. The HSOP's are currently published on paper, with no formal guidance on the structure: a 355-page handbook encodes all the 509 SOP's, and is revised every three years. HSOP's are being developed and maintained by teams belonging to the department to which the procedures apply; each department involves one or more different test- and examination providers, i.e., laboratories and services. HSOP's are approved by the Head of the department; once approved, they are submitted for publication to the Health Care Direction; at this stage minor adjustments may be done, but the procedure may be also sent back to the department.

By analysing the handbook in collaboration with medical experts, we discovered that HSOP's were not all structured in the same way, but they shared common elements and terms. We normalized some terms, and created a sort of superset embedding the various elements found in the procedures and considered necessary by the medical experts.

To formally describe the model we followed the guideline modelling approach of GEM [8]: thus it has been encoded with a XML-Schema, and the HSOP's encoded with XML. It should be stressed that so far the HSOP's were developed with no underlying model, thus were not standardized on a unique set of terms and entries. This adds value to our preliminary modelling work, because medical experts established a common structure and terminology to be adopted.

\subsection{The Logical Scheme of the HSOP's}

Two classes of HSOP's have been identified: the Laboratory test and Diagnostic test, which showed enough differences to be managed separately. The former is composed of 13 elements, the latter of 18.

The model includes a header, whose entries come from the Dublin Core metadata initiative [14], and a body, which includes all the entries found in 
the original procedures that were considered mandatory by the medical experts. The header is common to both HSOP classes; the differences arise in the body.

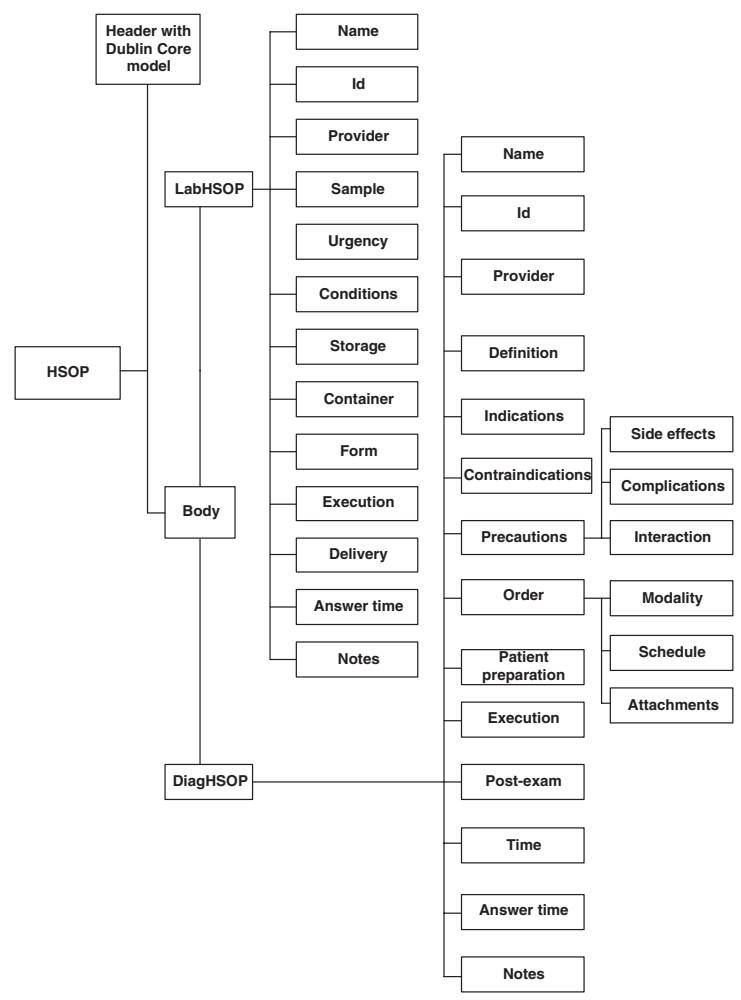

Fig. 1. The HSOP logical scheme

Part of the procedures have been manually converted to XML following the scheme, for testing purposes, but in the automated system (see the next section) a markup helper supports the human operator in correctly encoding all the available procedures.

\section{HOPERA: An Authoring and Workflow System}

A document workflow model was also developed, based on the three-step process described in the previous section.

Four categories of users have been considered with different privileges on the HSOP management. All the hospital staff members may read the procedures already published, but do not take part in the workflow process. Working group members may read, edit and create new procedures, as well as pass them to 
the Head of the department; the latter may read, edit and pass them back and forth; at the topmost level, the Health Care Director may read, edit, pass back or publish the procedures. In this way, publishing a HSOP undergoes three steps, each one regarding a category of operators/users; at each step the XML document is marked with the appropriate tags (figure 2).

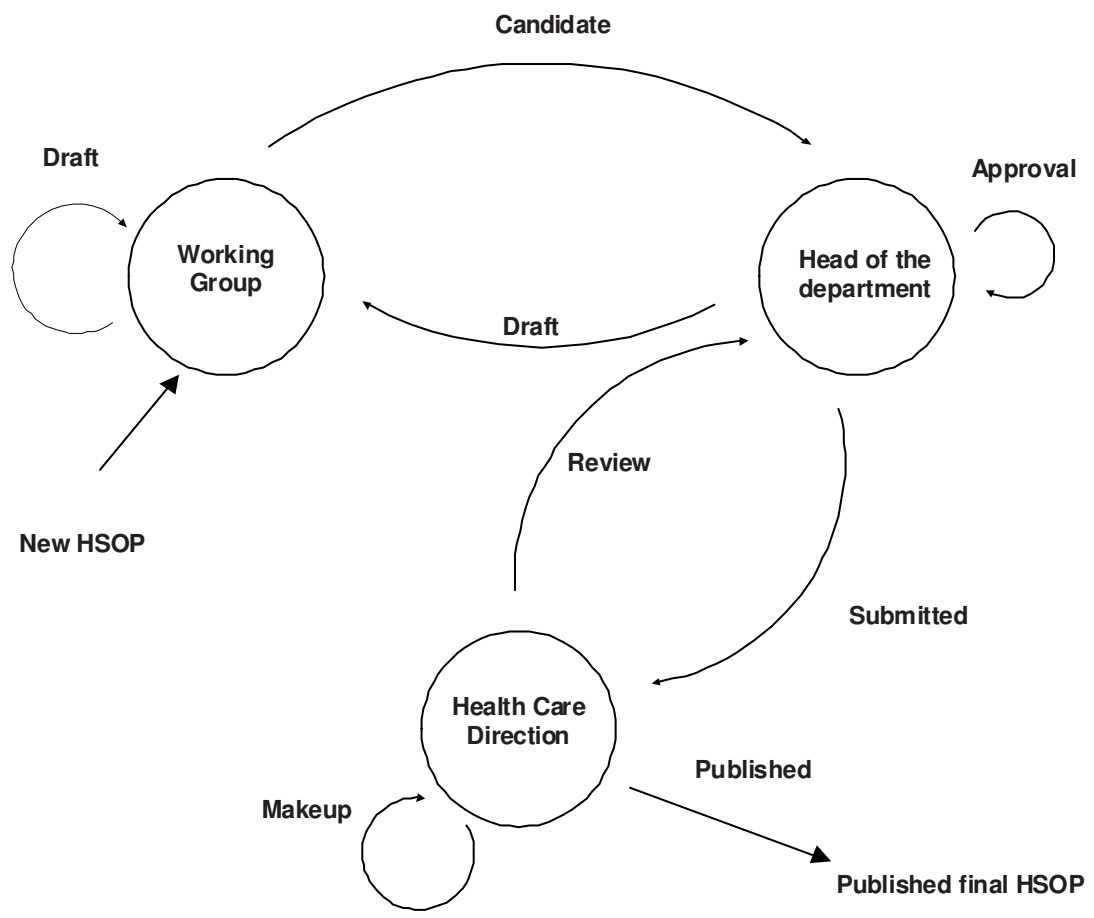

Fig. 2. The HSOP workflow model and the associated tags

A new software system called HOPERA (Hospital Operating Procedures Editing, Retrieval and Administration) has been designed for HSOP authoring and workflow management purposes. Both functionalities are deeply intertwined: the HSOP authoring is a kind of social interaction that HOPERA is intended to support. The system adopts an information-centered approach to authoring, since the latter is grounded on a well-defined model of pre-existing information. Authored HSOP's are also given an archive to be stored and retrieved for subsequent use, with versioning capabilities. 


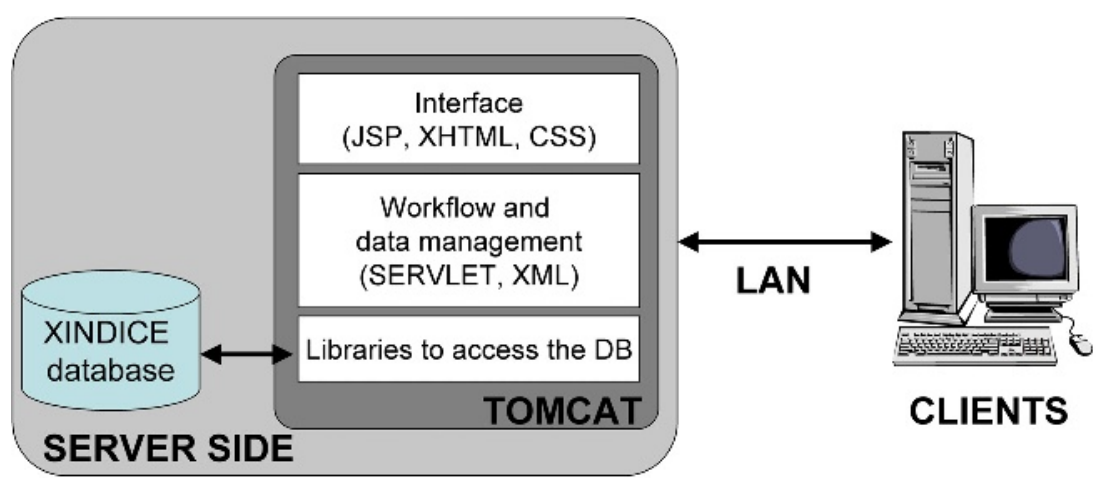

Fig. 3. Architecture of HOPERA

\subsection{HOPERA: System Architecture}

HOPERA is basically a web application implemented on an open-source platform, and enables the network access by multiple users. The system is composed by three main blocks (figure 3): the user interface, the server-side software modules, and the HSOP database.

The first two blocks have been implemented adopting the Java Server Pages (JSP) and Servlet technologies, which are popular for web applications. In particular, the user interface has been implemented adopting the standard XHTML and the style sheets (CSS). The HSOP database has been realised by means of the XML-native open-source database Xindice (Apache Software Foundation). Within this kind of database, XML documents are indexed as the basic logical units and grounded on the concept of Collection, a well-defined group of documents. In our implementation, one collection has been created, with two subcollections: one containing all documents of the type 'operating procedure'(the HSOP's), the other storing all user accounts.

\section{Using HOPERA}

The access to HOPERA is through a page that performs the user authentication and classification. The home page is then generated dinamically according to the user category. From that page, users may access, create or modify SOPs.

\subsection{Knowledge Access}

The hospital staff members may perform query and retrieval actions on the HSOP knowledge base, searching it by letter and by field value on the metadata. Once retrieved a list of browsable HSOPs, a click on the requested HSOP causes the visualization of its content, as in figure 4 Further reference information is available via hypertextual links, e.g., to the protocols governing some specified 


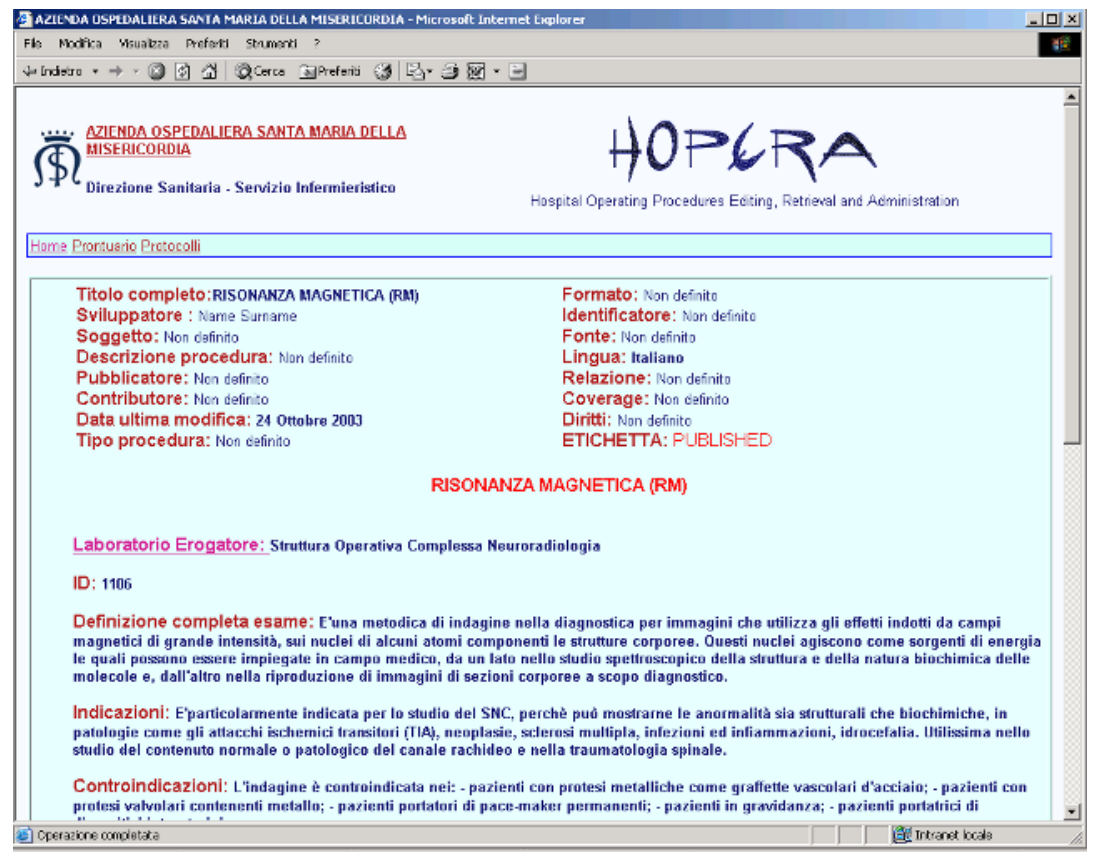

Fig. 4. Visualization of the HSOP concerning the NMR examination

actions, the departments and individual operators involved. Being HOPERA a web-based system, the access may occur from any node of the Hospital Intranet, and in particular from the nurses computers, which are the main users of HSOPs. A typical scenario could be that of a nurse needing to book an NMR examination for a patient: he/she can access the suitable SOP by searching for NMR, then browsing the retrieved information to have guidance in pre-examination patient preparation, eventually checking for contraindications and drug interaction related to the specific patient. There he/she can also find how to deal with the post-examination follow-up, if needed. Of course, the experienced nurse will not need to check all SOPs every time, while young nurses, or even nurses tranferred in a new ward, may use HOPERA as a source of either operational knowledge and learning material. This is thus a support to socialization and internalization of knowledge.

\subsection{Knowledge Creation}

Users of a working group can choose among three options, two of which are creation and modification of HSOPs. The interface provides them with a markup helper to correctly encode the procedure with respect to the model. Modification occurs only among the list of HSOPs still to be published, which includes either procedures to be corrected after examination by the Head of Department or 
Health Care Direction, or procedures to be examined in a cyclic process of revision, where recombination occurs of the previous version and external knowledge in the form of novel research results, policies, etc.

In the revision phase, interesting contributions might be provided by users, which are commonly excluded from the HSOP creation process: they can discover problems occurring during execution, errors, unnotified changes in the real procedure in respect to the modelled one.

In order to exploit these contributions, the most important development we are planning for HOPERA is an annotation system. Every user will be able to annotate HSOP's with personal comments, in order to notify problems encountered following the procedure, or suggested improvements. Working groups may then access to the annotations and take them into account when revising the HSOP's. This is a way for supporting knowledge socialization and externalization. In order to implement user annotations, we are planning to use the Annotea technology [17], which is provided by the W3C Consortium. Annotea allows for metadata-based annotations attached to documents or parts of documents, using web technologies.

\section{Conclusions and Beyond...}

The main contribution of the present paper is a model of the knowledge involved in the development of standard operating procedures in a health care organization. The main objects of interest, the HSOP's, have been given an abstract representation encoded as an XML-schema; the procedures themselves have been encoded as XML documents, and archived by means of an open source, XML-based archive. The dynamics of the HSOP development process has been captured by a workflow scheme. All models concurred to the design of an automated system, HOPERA, which is intended to support the information flows that have been identified.

An interesting scenario has been put forward and simulated by the automated system: that of knowledge creation within a health care institution. The idea has been verified of a dynamic process of social interaction, involving both explicit and tacit knowledge in a sort of continuous cycle, in which socialization, externalization, recombination and internalization steps are at work. Learning is ubiquitous in such a process and might be even identified with the knowledge creation itself, in such a way that the concept of organizational learning can be given a procedural sense.

HOPERA is being experimented inside the Udine City Hospital Intranet system, which currently provides knowledge management services [16].

Work is still in progress on HOPERA and further developments are planned in the near future, in addition to the annotation system described in the previous section. The most relevant one is extending the system functions with e-learning capabilities, i.e., a way to boost and make more explicit the distribution of knowledge within the organization. The same HSOP models will be adopted 
as courseware schemes to design training activities and repositories of learning objects for the staff involved.

By correctly characterizing HSOP's through metadata, and in particular by using controlled vocabularies and terminologies like ICD9-CM and SNOMED, it is possible to unambiguously associate them to specific health care conditions and treatments. This may be applied not only in the passive way we are currently pursuing, i.e., through a procedure browser, but also by active connections with the electronic health care record system. In fact, we may suppose that, when the physician orders a laboratory test or a diagnostic procedure (through an encoded term), the corresponding HSOP (or HSOP's) are automatically recalled, with easier and faster decision making. A similar support could be provided for facilitating the access to relevant scientific literature.

Finally, the same, ubiquitous XML allows to maintain multilingual versions of the HSOP's, thus enabling further contacts and exchanges among health care Institutions in different Countries.

\section{References}

1. M. Stefanelli: The socio-organizational age of artificial intelligence in medicine, Artificial Intelligence in Medicine 2001; 23: 25-47

2. Nonaka I., Takeuchi H.: The knowledge-creating company, Oxford University Press, Oxford, 1995.

3. Argyris C., Schön D.: Organizational learning II, Addison-Wesley, London, 1996

4. M.J.Field, K.N.Lohr (Editors): Clinical Practice Guidelines: Directions for a New Program, Institute of Medicine, 1990. http://www.iom.edu

5. G. Saadawi: An XML syntax for clinical laboratory procedure manuals, 7 th Annual Congress on Advancing Pathology Informatics, Imaging and the Internet, Pittsburgh, USA, 2002. http://www.pathology.pitt.edu/apiii02/Sci-Saadawi.htm

6. M.Hoenicka. The SOP DTD, http://ourworld.compuserve.com/homepages/hoenicka_markus/sopdtd.html

7. Shiffman R.N., Karras B.T., Agrawal A., Chen R., Marenco L., Nath S.: GEM: A proposal for a more comprehensive guideline document model using XML Journal of American Medical Informatics Association 2000;7(5):488-498

8. Shiffman R.N., Agrawal A., Deshpande A.M., Gershkovich P.: An approach to guideline implementation with GEM. Medinfo 2001;10(Pt 1):271-5

9. G. Hripcsak. The Arden Syntax for medical logic modules: introduction, Computers in Biology and Medicine 1994;24(5):329-30.

10. Shahar, Y.; Miksch, S.; Johnson, P.: The Asgaard Project: A Task-Specific Framework for the Application and Critiquing of Time-Oriented Clinical Guidelines, Artificial Intelligence in Medicine, 14, pp. 29-51, 1998.

11. V. L. Patel, T. Branch, D. Wang, M. Peleg, A. A. Boxwala: Analysis of the Process of Encoding Guidelines: An Evaluation of GLIF3, Methods of Information in Medicine 2002; 41(2): 102-113.

12. Tu, S.W., Musen, M.A, The EON Model of Intervention Protocols and Guidelines. 1996, Proc. of 1996 AMIA Annual Fall Symposium, Washington DC, pp. 587-591.

13. P. Mika, V. Iosif, Y. Sure, H. Akkermans. Ontology-based Content Management in a Virtual Organization, In Handbook on Ontologies in Information Systems, S. Staab, R. Studer (eds.), Springer Series: International Handbooks on Information Systems, 2003. 
14. The Dublin Core Metadata Initiative: http://www.dublincore.org/

15. The Apache Xindice web site: http://xml.apache.org/xindice/

16. The Udine City Hospital Knowledge Centre: http://www.ospedaleudine.it/kc

17. J.Kahan, M.R.Koivunen, E.Prud'Hommeaux, and R.R.Swick. Annotea: An Open RDF Infrastructure for Shared Web Annotations. in Proc. of the WWW10 International Conference, Hong Kong, May 2001. 